3

4

5 8

\title{
An exploratory analysis of variations in quiet eye duration within and between
} levels of expertise

\author{
Shannon Jingyi Chia
}

Physical Education \& Sports Science, Nanyang Technological University, Singapore Jia Yi Chow

Physical Education \& Sports Science, Nanyang Technological University, Singapore Masato Kawabata

Physical Education \& Sports Science, Nanyang Technological University, Singapore Matt Dicks

Department of Sport and Exercise Science, University of Portsmouth, United Kingdom Marcus Lee Singapore Sports Institute, Singapore

Accepted: International Journal of Sport and Exercise Psychology, $13^{\text {th }}$ September 2015

Correspondence should be addressed to: Shannon Jing-Yi Chia, Physical Education and Sports Science, National Institute of Education, Nanyang Technological University, 1 Nanyang Walk, Singapore 637616. Telephone: +65 97948535. Email:

chia0289@e.ntu.edu.sg 


\section{Abstract}

The ability to pick-up task relevant visual information during movement control is crucial in successful sport performance. Quiet eye $(\mathrm{QE})$, the final fixation prior to final movement onset, has been shown to be characteristic of the visual search strategies exhibited by skilled athletes in self-paced aiming tasks. Longer QE durations were previously associated with skill and successful performance outcomes. In this study, gaze behaviour data of six expert $(\mathrm{E})$ and six novice $(\mathrm{N})$ ten-pin bowlers were measured using a mobile eye tracker as they completed 20 trials of two single-pin conditions each (Easy: 1-pin; Hard: 10-pin). Expert bowlers exhibited significantly longer QE durations in both conditions as compared to their less skilled counterparts. However, QE duration was not found to be significantly different as a function of accuracy nor task condition. Further detailed analysis revealed considerable variance in QE characteristics between individuals, warranting the need to explore individualized interventions centered on the development of perceptual-motor control during selfpaced aiming tasks. Moreover, this study raised an important methodological issue relating to the analysis of trials with the absence of QE. 


\section{Introduction}

Sports expertise, defined as the ability to consistently demonstrate superior athletic performance (Starkes \& Ericsson, 2003), has been of great interest to researchers and practitioners. Experts, unlike novice performers, possess enhanced perceptual-motor skills, which are often underpinned by differences in gaze control strategies (Mann, Williams, Ward, \& Janelle, 2007). Specifically, the environment consists of both variant and invariant information, with accurate perceptual-motor behaviour emerging as performers learn to exploit and calibrate their movements to the invariant information (van der Kamp, Rivas, van Doorn, \& Savelsbergh, 2008). Successful performance is therefore underpinned by an individual's ability to direct gaze to task-relevant invariant information in the environment during movement control (Williams, Davids, \& Williams, 1999).

The vision-in-action (VIA) paradigm (for a review, see Vickers, 2007) has been used to examine the gaze control strategies that underpin expert movement control in real time. Vickers (1992) reported that skilled golfers exhibited a distinct visual search strategy characterized by a fixation on the back of the ball for a mean duration of two seconds prior to, and following ball contact during successful golf putting. This gaze pattern termed the Quiet Eye (QE) (for reviews, see Vickers, 2007; Vine, Moore, \& Wilson, 2014) is the final fixation on a location or target in the visuomotor workspace prior to the initiation of final movement onset. QE has been found to underlie expert performance across a wide variety of aiming tasks with longer QE periods believed to be associated with skill level and successful performances (e.g., Causer et al, 2010; Seung-Min, Seonnjin, \& Park, 2009; Wilson \& Pearcy, 2009). Specifically, QE was originally proposed to reflect the time needed for motor preparation within a predictive control strategy whereby movement parameters (e.g., direction) and limb timing/coordination are programmed and fine-tuned (Vickers, 1996). As such, longer QE periods extend the duration of this critical preparation period, translating to a 
1 higher likelihood of successful motor performance (Vickers, 2011). However, it should be

2 noted that this relationship between QE duration and performance is non-linear, such that

3 further increases in QE duration may not result in additional concomitant improvement in

4 performance (Moore, Vine, Cooke, Ring, \& Wilson, 2012). Instead, an optimal range of QE

5 duration may exists depending on the nature and specific motor task involved, with prolonged

6 durations associated with possible attentional and/or postural fatigue (Behan \& Wilson,

7 2008). The vast majority of these studies, however, have also reported QE as an average

8 duration between groups, with little consideration of any within-group variation. This

9 suggests the absence of an average, over-arching perceptual strategy that all individuals use

10 in order to achieve the same outcome. Given strong evidence on the reciprocal relation

11 between perception and action (van der Kamp et al., 2008); it is possible that individual differences reported in movement control despite achieving the same outcome, may be underpinned by variation in information pick-up. This is evidenced by between-participant differences in gaze strategies. Indeed, in a small number of existing studies, findings have revealed individual differences in gaze control during cricket batting (Croft, Button, \& Dicks, 2010), intercepting penalty kicks among experienced football goalkeepers (Dicks, Davids, \& Button, 2010) and football goalkeeping in deceptive kicks (Tay, Chow, Koh, \& Button, 2012). Even in the examination of sports expertise, individual variability in performance among skilled athletes seems to be a norm rather than an exception. For example, it was observed that even among expert soccer players, they exhibited different kicking patterns in a common kicking task (Chow, Davids, Button, \& Koh, 2006). Such observed individual variability in performance questions the value of over-emphasizing group comparisons. Rather, the focus should be on the individual performer. individual differences that may exist as a function of skill level (i.e., between experts and 
1 novices). One notable study by Mann and colleagues (2011) illustrated individual differences

2 in QE duration within both expert and near-expert golfers in a golf putting task based on

3 results from single-subject analysis. Here, variations in QE duration were observed between

4 golfers in each skill group who had similar numbers of hits and misses. As such, even without

5 differences in performance outcome, it is reasonable to expect differences in QE duration.

6 Within each sports task, the degree of task difficulty can vary depending on the

7 constraints imposed. For example, in an aiming task like bowling, the position of pins can

8 impact task complexity (Lasser, Borden, \& Edwards, 2006). A bowler gets two rolls at every

9 turn; a strike occurs if all 10 pins are knocked down on the first roll; a single pin spare

10 happens when the bowler hits 9 pins on the first and the last on his/her second roll

11 (Professional Bowlers Association, 2014). With single pin spares, certain pins are relatively more difficult to hit depending on the handedness and individual bowling style. For example, hitting the 10-pin, positioned at the extreme right corner, can be incredibly tough for a righthanded hook bowler (Forrest \& Iannucci, 2010). Variations in QE characteristics within and between skill levels may also be better assessed with different task conditions in terms of difficulty levels.

The present study examined the characteristics of QE in expert and novice bowlers in two task conditions. It was predicted that expert bowlers would exhibit longer QE durations compared with novices (see Mann et al., 2007). Furthermore, it was hypothesized that a within-group comparison might also reveal some of the key individual differences in QE among bowlers of the same skill level, which has not been adequately investigated in previous works. 


\section{$1 \quad$ Method}

\section{Participants}

3 A total of 12 bowling players participated in the present study. They were six (4 males and 2

4 females) expert bowlers (age: $18.0 \pm 1.55$ years) from the Singapore National Bowling Squad

5 and six (2 males and 4 females) novice bowlers without any competitive experience (age:

$623.0 \pm 1.67$ years). The novice bowlers were numbered Participant 1 to 6 ; experts were

7 numbered from 7 to 12 . The experts had averaged $7.67 \pm 1.86$ years of competitive

8 experience (e.g., Asian Bowling Championships, Commonwealth Bowling Championships,

9 Singapore National Championship, Southeast Asian Games, World Youth, and Asian Schools

10 Championships). The sample size was similar to previous studies examining visual behaviour and Quiet Eye (see Millslagle, Hines, \& Smith, 2013; Oliveira et al., 2008; Vickers, 1992).

All the participants were right-hand dominant and reported normal vision. The study was approved by an institutional review board and adhered to the guidelines for ethical practice. Participation was voluntary and informed consent was received from each participant.

Task

Participants were required to perform 10 trials for each of the two single pin conditions (1-pin and 10-pin) in a randomized order (see Figure 1). Depending on the constraints imposed, task conditions can be manipulated. In bowling, the task condition differs depending on the position of pins (Lasser, Borden, \& Edwards, 2006). Single pin spares happen when the bowler hits 9 of 10 pins on the first roll of his/her turn (Professional Bowlers Association, 2014). The 1-pin was chosen as regardless of the handedness of the bowler, the 1-pin forms 'part of the pocket' (Forrest \& Iannucci, 2010, p. 165), forming an angle that maximizes the chances of scoring strikes; between the 1- and 2-pin, 1- and 3-pin for left- and right-handed bowlers respectively (Fuss, 2009). The second task condition involves hitting the 10-pin which is positioned close to the gutter, a pin often hard for the bowler to hit successfully 
1 (Forrest \& Iannucci, 2010). Input from an expert bowling coach confirmed the validity of

2 task conditions. There were no restrictions on the starting position for the participants to

3 ensure that there is representativeness in the task design so that the participants can best

4 replicate their true performance and visual search behaviours for this study (see Pinder,

5 Davids, Renshaw, \& Araújo, 2011).

\section{Apparatus}

7 The experiment was performed on a well-conditioned bowling lane oiled to 38 feet, with

8 internationally accepted bowling balls and pins. Eye movement data were collected using an

9 Applied Science Laboratories (ASL) Mobile Eye-XG movement system, a video-based

10 monocular corneal-reflection system which measures point of gaze (at $30 \mathrm{~Hz}$ ) based on the

11 vertical and horizontal distances between the center of the pupil and corneal reflection after correcting for second-order effects. The system is accurate to within $0.5^{\circ}$ to $1^{\circ}$ of visual angle with a visual range of $60^{\circ}$ horizontal and $40^{\circ}$ vertical. In addition, a side-on digital video camera (Casio Exilim EX-FH 100) was used to determine point of ball release (at $30 \mathrm{~Hz}$ ). See Figure 2 for a diagrammatic representation of the experimental set-up.

\section{Procedure}

17 Participants were tested individually and were given 5 bowling frames for warm-up before the eye tracker was fitted. This was followed by 10 minutes of practice for familiarization. Participants bowled to a frame consisting of all 10 pins for both the warm-up and familiarization trials. With ball in hand, participants were instructed to look towards the clapperboard for a calibration check before each test trial. This also ensured that all participants started their aiming process at the same time. The clapperboard signalled the start of each trial, with ball release indicating the end. Prior to each trial, participants had to rate their perceived task difficulty and confidence. Participants were allowed to rest whenever necessary to minimize the effect of fatigue on performance. 
3 For each condition, individual performance outcome was obtained in terms of the percentage

4 of pins hit.

$5 \quad$ Quiet Eye $(Q E)$

6 QE was operationally defined as the final duration of fixation on a specific target before the 7 onset of movement time (Vickers, 1996). Movement time can be described as the portion of 8 an action 'common to all performers' (Harle \& Vickers, 2001, p. 290). In bowling, as in 9 many other discrete skills, preparatory actions can differ greatly between individuals. For 10 instance, some individuals especially the less skilled may not have a distinct backswing arm movement unlike that of skilled bowlers. Despite variability in these preparatory actions, the forward arm swing movement prior to ball release is common to all since the ball needs to be propelled towards the pins. It also does not seem to matter which target location is chosen as long as there is only one specific location where attention and gaze is directed towards (see Vickers, 2007).

With reference to the above points, QE was defined as the final fixation on a specific location for a minimum of $100 \mathrm{~ms}$ prior to the initiation of the forward arm swing movement in this study. A side-on camera was used to determine the first frame indicating forward arm movement of the individual. QE duration was compared between both skill-level groups (E vs N) across the two conditions (easy vs hard). Fixation duration for QE analyses was calculated automatically by the ASL software. Out of the 240 trials, no fixations were recorded for 11 trials (4.6\%). Interestingly, all 11 trials suggesting the absence of QE belonged to the novice group. Following the procedures reported in previous studies (see Nagano, Kato, \& Fukuda, 2006; Williams et al., 2002), a zero value was entered for trials without fixations. 
1 However, this procedure may appear erroneous as no fixations operationally identified

2 with the cut-off value (i.e., a minimum of $100 \mathrm{~ms}$ ) are not exactly identical to zero

3 value and consequently, replacing no fixation with a zero value positively skews the

4 distribution of data. Yet, there seems to be no consensus in the existing literature on

5 how to deal with trials with no fixations. Alternatively, some researchers have excluded

6 these trials from the analysis (see Klostermann, Kredel, \& Hossner, 2013). It would be

7 logical to consider trials where no fixations were captured due to technical difficulties

8 as invalid cases and exclude them from further data analysis. However, if no fixations

9 were caused by skill-related reasons (e.g. lack of skill), the absence of QE represents

10 important skill-related information that should not be ignored. Therefore, to address this

11 methodological issue, both methods for dealing with no fixation trials (i.e., zero value

12 replacement and trial exclusion) were adopted and the results of each method were

13 reported in this study.

14 Perceived task difficulty and confidence

15 Subjective ratings of task difficulty and confidence level were measured using two separate 16 single items, each rated on a 7-point Likert scale. The scale for task difficulty ranged from 1 17 (very easy) to 7 (very difficult) and the scale for confidence level ranged from 1 (not confident al all) to 7 (very confident). The differences between the two perceived scores (i.e., task difficulty - confidence) were calculated for each trial (Kawabata \& Mallett, 2011) and then, the mean of the difference for 10 trials was produced for each participant for each task condition. 


\section{Data Analysis}

2 A 2 (skill: expert, novice) $\times 2$ (task condition: easy, hard) analyses of variances

3 (ANOVA) was conducted on QE and performance data independently to examine the

4 two main effects and one interaction effect of contextual factors on QE and

5 performance each. To explore whether there were differences in perceived task

6 difficulty and confidence levels between groups and conditions, a 2 (skill: expert,

7 novice) $\times 2$ (task condition: easy, hard) was also conducted on the difference between

8 the two perceived scores.

\section{$9 \quad$ Results}

\section{Bowling Performance}

Descriptive statistics are presented in Table 1. A 2 (skill: expert, novice) $\times 2$ (task condition: easy, hard) (ANOVA) on the percentage of successful performance revealed a significant main effect for skill, $F(1,10)=110.56, p<.001, \eta_{\mathrm{p}}{ }^{2}=.974$. Experts' successful performance rates $(86.7 \pm 4.1 \%)$ were significantly higher compared to that of the novices $(25.8 \pm 4.1 \%)$.

There was no significant main effect for condition, $F(1,10)=2.738, p=.129, \eta_{\mathrm{p}}{ }^{2}=.215$, and no interaction effect between skill level and task condition, $F(1,10)=1.109, p=.317, \eta_{\mathrm{p}}{ }^{2}=$ .100 .

\section{Quiet Eye}

A 2 (skill: expert, novice) $\times 2$ (task condition: easy, hard) ANOVA was performed on QE duration. As mentioned earlier, two different methods were used to deal with the no-fixation trials for calculating QE duration. Descriptive statistics of the two groups' QE duration are presented in Table 2, and results for the respective methods are presented below. Zero value method. A significant effect for skill, $F(1,10)=5.110, p=.047, \eta_{\mathrm{p}}{ }^{2}=.338$ was found. Experts exhibited on average, significantly longer QE durations as compared to the novices for both the easy (E: $196.33 \pm 50.53 \mathrm{~ms}$; N: $165.00 \pm 32.78 \mathrm{~ms})$ and hard (E: 
$1 \quad 194.33 \pm 43.63 \mathrm{~ms} ; \mathrm{N}: 149.33 \pm 31.01 \mathrm{~ms}$ ) conditions. However, no significant effect for

2 condition, $F(1,10)=0.305, p=.593, \eta_{\mathrm{p}}{ }^{2}=.030$ nor significant interactions were found, $F(1$,

$3 \quad 10)=0.182, p=.678, \eta_{\mathrm{p}}{ }^{2}=.018$.

4

Trial exclusion method. Experts exhibited on average, longer QE durations as compared to the novices for both the easy (E: $196.33 \pm 50.53 \mathrm{~ms} ; \mathrm{N}: 182.92 \pm 23.24 \mathrm{~ms})$ and hard (E: $194.33 \pm 43.63 \mathrm{~ms} ; \mathrm{N}: 163.14 \pm 26.66 \mathrm{~ms})$ conditions. ANOVA revealed no significant main effects for skill $\left(F[1,10]=2.574, p=.140, \eta_{\mathrm{p}}{ }^{2}=.205\right)$, condition $(F[1,10]=$ $\left.0.420, p=.532, \eta_{\mathrm{p}}{ }^{2}=.404\right)$ and no significant interaction effect $(F[1,10]=0.280, p=.608$, $\left.\eta_{\mathrm{p}}{ }^{2}=.027\right)$. Interestingly, the significant main effect for skill which was found with the zerovalue replacement method was no longer observed with the trial exclusion method. Individual differences. To elaborate skill-related information of QE behaviour, QE duration based on the zero-value replacement method was closely examined further. It revealed some interesting findings pertaining to individual differences. Variations in QE duration amongst individuals with the same performance scores in their respective skill group were evident for each condition. Trials with the absence of QE (easy: 6 trials; hard: 5 trials) were represented graphically as a data point of zero. In the easy condition, this variation can be observed between novice Participants 4 and 6 as well as expert Participants 8, 10 and 11 (see Figure 3a). It seems that despite achieving the same level of performance scores, there was no clear indication of an optimal QE duration associated with these scores. Likewise, even though four out of the six participants in each skill group (novice Participants 3, 4, 5 and 6; expert Participants 7, 8, 11 and 12) scored the same scores for the hard condition, their QE durations varied across a wide spectrum of values (see Figure $3 b$ ).

In addition, participants with similar QE duration had drastically different performance scores. For instance, average QE duration for novice Participants 1 and 3 were $147.00 \pm 113.63 \mathrm{~ms}$ and $148.00 \pm 112.72 \mathrm{~ms}$ respectively in the easy condition. Yet, 
1 Participant 1 managed to hit the pin only once unlike Participant 5 who hit the pin in five of

2 the 10 trials. This was also observed amongst the experts with skilled Participant 7 exhibiting

3 a mean QE duration of $190.00 \pm 102.74 \mathrm{~ms}$ and a performance score of $60 \%$ whilst

4 Participant 8 had a 100\% success rate even though mean QE duration was $195.00 \pm 88.60 \mathrm{~ms}$.

$5 \quad$ Individual performance scores are illustrated on Figure 4 for reference.

$6 \quad$ Associations between $Q E$ and performance outcome

7 The associations between QE and performance outcome (hits and misses) were examined.

8 However, there was a lack of unsuccessful (missed) shots for both conditions for the experts

9 (easy condition: three participants; hard condition: one participant). Therefore, $t$-test was

10 conducted on performance scores (easy: $88.3 \pm 16.0 \%$; hard: $85.0 \pm 8.4 \%$ ) and no significant

11 differences were observed between conditions $(t[5]=1.96, p=.107, d=1.76)$ for the experts.

12 It is possible that the task manipulation for the experts was not as successful (further

explanation will be made in the discussion section). Therefore, for more meaningful

interpretation, analyses of the relationship between QE and performance outcome (hits and misses) was only performed for the novices. All trials from the novices were included for this analysis. Descriptive statistics of their QE duration are presented in Table 2. Unexpectedly, the longest and shortest QE duration were observed for successful performances in the easy and hard conditions, respectively. A one-way repeated ANOVA was conducted on QE duration to examine whether QE duration was different between hits and misses for each condition for the novices. Here, differences in QE duration with regards to performance outcome for both the easy and hard conditions approached significance, $F(3,15)=2.950, p=$ $.067, \eta_{\mathrm{p}}^{2}=.371$.

Easy condition. Based on their performance scores, Participants 3 and 5 had the most number of successful shots (hits) as compared to the other novices. Interestingly, while it seems like the successful shots executed by Participant 3 were accompanied by longer QE 
1 duration in comparison to unsuccessful trials, QE durations for both outcomes were relatively

2 similar for Participant 5. Secondly, contrary to initial predictions, unsuccessful shots (misses)

3 by Participant 4 were characterized by longer QE durations as compared to the successful

4 shots. That is, increased QE duration was also considered to be associated with poor

5 performance amongst some of the novice bowlers in this study (see Figure 5a).

$6 \quad$ Hard condition. Longer QE durations for successful shots as compared to

7 unsuccessful ones were observed for Participants 1 and 4. However, it seems like longer QE

8 durations in the hard condition did not translate to better outcomes. Specifically, the

9 unsuccessful shots by Participants 3, 5 and 6 were clearly associated with longer QE

10 durations (see Figure 5b).

Perceived task difficulty and confidence level

Descriptive statistics of perceived task difficulty and confidence levels as well as their difference are presented in Table 3. Experts' confidence score was higher than their perceived task difficulty score across the two task conditions, whereas novices showed the opposite pattern. That is, novices' perceived task difficulty score was higher than the confidence score in both task conditions and the difference score in the hard condition was slightly larger than that in the easy condition.

A 2 (skill: expert, novice) $\times 2$ (task condition: easy, hard) ANOVA revealed that novices' difference scores were significantly higher than experts' scores, $F(1,10)=$ $139.06, p<.001, \eta_{\mathrm{p}}{ }^{2}=.900$. However, the main effect of task conditions $(F[1,10]=$ $\left.0.67, p=.434, \eta_{\mathrm{p}}{ }^{2}=.062\right)$ and the interaction effect of skill level and task conditions $\left(F[1,10]=1.80, p=.210, \eta_{\mathrm{p}}{ }^{2}=.153\right)$ were found non-significant. These results indicated that experts' confidence to perform the task successfully over their perception of the task difficulty was significantly higher than novices across the task conditions. 
1 On the other hand, novices perceived each task quite difficult beyond their confidence

2 level.

\section{Discussion}

4 The purpose of this study was to examine differences in QE characteristics between expert

5 and novice ten-pin bowlers under two different task conditions. Experts were predicted to

6 exhibit longer QE duration compared to the novice bowlers. It was also expected that

7 successful shots (hits) would be associated with longer QE durations. Furthermore, individual

8 differences in visual behaviour might be evident even amongst bowlers of the same skill

9 levels.

10 Quiet Eye (QE)

11 Duration of QE supports the existing literature (e.g., Causer et al, 2010; Seung-Min, Seonnjin, \& Park, 2009) with experts exhibiting significantly longer QE durations compared to novices, providing support for QE being a discriminating characteristic of sport expertise. The analysis of QE duration, however, raised an important methodological issue relating to the treatment of trials without any fixations. Specifically, in this study, QE was absent in 11 trials belonging to the novice bowlers. While this in itself is clearly a discriminating factor of expertise, past studies have reported that these were excluded entirely from the analysis (Klostermann, Kredel, \& Hossner, 2013). This has strong implications on the subsequent results reported. Alternatively, some had chosen to use a zero value to represent these trials (Nagano et al., 2006; Williams et al., 2002). While a major criticism in this is the skewing of data set which leads to biased mean values, the same can be argued for trials with exceptionally long QE durations (e.g. Participants 7 and 11 as reflected in Figure 3b). Should these trials then be removed as well? In this case, the selection of method to adopt proved to be critical as the results differed greatly as presented in the earlier sections. Therefore, there are important methodological issues that need to be addressed in the QE literature. 
2 2000; Williams et al., 2002), QE duration was not found to be significant as a function of accuracy nor condition under both zero-value and exclusion methods of analysis.. As described by Vickers (1996), longer QE periods are believed to support skilled performance as they enable extended periods of movement programming prior to action execution. As such, due to its position and the handedness of participants, striking the 10-pin as opposed to 1-pin would require more parameters to be processed by the visuomotor system. Accordingly, one would expect longer QE duration in tasks that are more difficult (see Williams et al. 2002). However, this difference was not observed for this study. significant difference in QE duration between different golf putting of different slope characteristics. The failure to find significant differences in QE durations between performance outcomes as well as task conditions could be due to the fact that single-pin spares in general, regardless of pin position, can be hit confidently and successfully by most expert bowlers. Although performance scores for the hard condition was lower than that of the easy condition for both skill groups, the difference in scores between each condition for both groups did not differ significantly (see Table 1). That is, experts performed equally well for both conditions whereas novices had low scores for both. A possible reason could be due to task familiarity and deliberate practice by the experts. As relatively few frames in a game have strikes, spares become very important for a bowler's success; making it a crucial part of their training programme (Wiedman, 2006). Contrastingly, novices probably have had little or no experience with dealing with single pin spares, making both conditions equally difficult. In addition, despite the participants rating the difficulty levels for each condition differently, their levels of perceived confidence could have affected the eventual results. In 
1 both conditions, it was found that experts' confidence score was higher than their perceived

2 task difficulty while the opposite was true for novices. Self-efficacy which refers to the

3 athlete's belief in their ability to perform the sport task, has been identified as one of the most

4 important variables related to sporting performance as well as a distinguishing factor for the

5 highly successful and less successful athletes (Beatty, Fawver, \& Janelle, 2015). Besharat and

6 Pourbohlool (2011) examined the moderating effects of self-efficacy on competitive anxiety

7 and subsequent sporting performance. Results from the study confirmed the moderating role

8 of self-efficacy, indicating that higher levels of self-efficacy decreased the negative

9 associations with any form of anxiety which would lead to better performance. In this case, any anxiety due to the test or task environment could have been moderated by the high levels of self-efficacy demonstrated by the experts. On the other hand, the confidence level of novices for both conditions was below that of the task difficulty. As such, the level of selfefficacy of bowlers could have had strong implications on the observed performance scores and QE characteristics. Results from this study suggests that the potential for QE as a discriminating factor for successful performance may be highly task specific and definitely requires further investigation. Moreover, where expert bowlers are involved, the combination of single and split conditions such as the 3-6-10 split, might shed further insights on interesting visual strategies pertaining to the role of QE employed for a bowling task.

Furthermore, inter-individual comparisons of QE data amongst the participants also cast some doubt on the purported anchoring role of QE in target aiming tasks. For instance, the exaggerated period of QE ( $\geq 400 \mathrm{~ms}$ ) (e.g., exhibited by novice Participants 4 and 2 ) in the easy and hard condition respectively did not prove to be beneficial in terms of performance outcomes. Here, it is possible that there may be variation in a 'necessary' QE duration for successful performance in different sports/tasks (Oliveira et al., 2008) or perhaps 
1 of attentional or postural fatigue whereby exceeding the optimal durations of QE may in turn

2 be counterproductive, affecting performance (Behan \& Wilson, 2008; Moore et al., 2012).

This has important implications on designing intervention programmes to enhance the use of QE and also how QE data could be interpreted as a function of task and performance context. As such, further research is warranted to validate the role of $\mathrm{QE}$ in aiming task and particularly bowling. Moreover, as discussed earlier, the relatively longer movement time for the bowling task may render QE to be less relevant. This has implications on other interceptive task with longer movement time such as kicking in a soccer penalty shot or even basketball free throw as previously mentioned.

Collectively, two interesting possible points can be concluded: First, it appears that the relative importance of QE in performance success is very much dependent on the task and movement of the individual. Secondly, the lack of significant differences in QE duration between successful and unsuccessful outcomes seems to suggest a limitation in using QE to predict performance success in certain sporting tasks. Typically, researchers interested in visual search behaviour in sport adopt the customary approach in comparing point-of-gaze across different skill levels (expert vs. novice in this case) in order to determine skill-based differences in visual search strategies. However, researchers have not adequately examined whether different visual search strategies (in this case, relating to QE) are present within a group where the participants are presumed to have a similar level of expertise.

Indeed, skilled performance may not only be due to differences in gaze strategies per $s e$, but also due to the relation between movement capabilities and information pick-up (Dicks et al., 2010; Tay et al., 2012). This result further augments the need for not just grouplevel analyses, but inter-individual analysis within groups. Therefore, while traditional perceptual training interventions often point to purported optimal strategies (e.g., QE), it appears that there may be no one best gaze training strategy for some tasks. Thus, the 
1 prominent role accorded to QE for proficient performance in aiming tasks may need to be re-

2 examined to determine its impact under various task and skill contexts as well individual

3 performer constraints.

\section{Conclusion}

5 A novel contribution with regards to this study was to examine QE duration between and

6 within levels of expertise in ten-pin bowling. Specifically, longer QE duration discriminated

7 the expert from novice bowlers. However, apart from group differences, considerable

8 variance in individual QE duration within-groups was observed. This confirms the need for a

9 shift towards more inter- and intra-individual analysis. Results from this study also revealed possible limitations in using $\mathrm{QE}$ as a performance indicator depending on the nature of the task and individual movement characteristics. As such, further research examining QE in more complex tasks (e.g., split conditions) as well as individual variations are warranted to advance our understanding of the role of QE in interceptive tasks such as bowling. This paper also raised an important methodological issue relating to the treatment of trials in which $\mathrm{QE}$ was absent. Currently, there are no consensus on how these trials should be analysed, questioning the accuracy of existing findings. The failure to address this has strong implications on QE research within the expert-novice paradigm. In conclusion, findings from this study extend the existing literature in our understanding of QE in impacting performance, offering promise for evidence-based approaches in future visuomotor skill training interventions. 


\section{$1 \quad$ References}

2 Beatty, G., F., Fawver, B., Janelle, C.M. (2015). Psychological determinant of expertise: Emotional reactivity, psychological skills, and efficacy. In J. Baker \& D. Farrow, Routledge handbook of sport expertise (pp. 245-258). New York: Routledge.

Behan, M., \& Wilson, M. (2008). State anxiety and visual attention: The role of the quiet eye period in aiming to a far target. Journal of Sports Sciences, 26(2), 207-215. doi: $10.1080 / 02640410701446919$

Besharat, M. A., \& Pourbohlool, S. (2011). Moderating effects of self-confidence and sport self-efficacy on the relationship between competitive anxiety and sport performance. Psychology, 2(7), 760-765. doi:10.4236/psych.2011.27116

Causer, J., Bennett, S., Holmes, P., Janelle, C., \& Williams, A. (2010). Quiet eye duration and gun motion in elite shotgun shooting. Medicine and Science in Sports and Exercise, 42(8), 1599-1608. doi: 10.1249/MSS.0b013e3181d1b059

Chow, J., Davids, K., Button, C., \& Koh, M. (2006). Organization of motor system degrees of freedom during the soccer chip: An analysis of skilled performance. International Journal of Sport Psychology, 37(2/3), 207-229.

Croft, J. L., Button, C. C., \& Dicks, M. (2010). Visual strategies of sub-elite cricket batsmen in response to different ball velocities. Human Movement Science, 29(5), 751-763. doi: 10.1016/j.humov.2009.10.004

Dicks, M., Davids, K., \& Button, C. (2010). Individual differences in the visual control of intercepting a penalty kick in association football. Human Movement Science, 29(3), 401-411. doi:10.1016/j.humov.2010.02.008

Forrest, A.J., \& Iannucci, L. (2010). Bowling for dummies. Hoboken, NJ: Wiley Publishing, Inc. 
1 Fuss, F. K. (2009). Design of an instrumented bowling ball and its application to performance analysis in tenpin bowling. Sports Technology, 2(3-4), 97-110.

Harle, S. K., \& Vickers, J. N. (2001). Training quiet eye improves accuracy in the basketball free throw. Sport Psychologist, 15(3), 289-305.

Janelle, C. M., Hillman, C. H., Apparies, R. J., Murray, N. P., Meili, L. L., Fallon, E. A., \& Hatfield, B. D. (2000). Expertise differences in cortical activation and gaze behavior during rifle shooting. Journal of Sport \& Exercise Psychology, 22(2), 167-182.

Kawabata, M., \& Mallett, C. J. (2011). Flow experience in physical activity: Examination of the internal structure of flow from a process-related perspective. Motivation and Emotion, 35(4), 393-402. doi:10.1007/s11031-011-9221-1

Klostermann, A., Kredel, R., \& Hossner, E. (2013). The 'quiet eye' and motor performance: Task demands matter!. Journal of Experimental Psychology: Human Perception and Performance, 39(5), 1270-1278. doi:10.1037/a0031499

Lasser, E.S., Borden, F., \& Edwards, J. (2006). Bowling: The Handbook of Bowling Psychology. West Virginia University: Fitness Info Tech.

Mann, D. Y., Coombes, S. A., Mousseau, M. B., \& Janelle, C. M. (2011). Quiet eye and the Bereitschaftspotential: visuomotor mechanisms of expert motor performance. Cognitive Processing, 12(3), 223-234. doi:10.1007/s10339-011-0398-8

Mann, D. Y., Williams, A., Ward, P., \& Janelle, C. M. (2007). Perceptual-cognitive expertise in sport: A meta-analysis. Journal of Sport \& Exercise Psychology, 29(4), 457-478.

Millslagle, D.G., Hines, B.B., \& Smith, M.S. (2013). Quiet eye gaze behavior of expert, and near-expert baseball plate umpires. Perceptual and Motor Skills, 116(1), 69-77.

Moore, L. J., Vine, S. J., Cooke, A., Ring, C., \& Wilson, M. R. (2012). Quiet eye training expedites motor learning and aids performance under heightened anxiety: The roles of response programming and external attention. Psychophysiology, 49(7), 1005-1015. 
1 Nagano, T., Kato, T., \& Fukuda, T. (2006). Visual behaviors of soccer players while kicking with the inside of the foot. Perceptual and Motor Skills, 102(1), 147-156.

Oliveira, R. F. D., Oudejans, R. R. D., \& Beek, P. J. (2008). Gaze behavior in basketball shooting: Further evidence for online visual control. Research Quarterly for Exercise and Sport, 79(3), 399-404. doi:10.1080/02701367.2008.10599504

Pinder, R., Davids, K., Renshaw, I., \& Araújo, D. (2011). Representative learning design and functionality of research and practice in sport. Journal of Sport and Exercise Psychology, 33, 146-155.

Professional Bowlers Association. (2014). Bowling Rules. Retrieved June 20, 2015 from http://www.pba.com/Resources/Bowling101/

Starkes, J. L., \& Ericsson, A.K. (2003). Expert performance in sports: Advances in research on sport expertise. Champaign, IL: Human Kinetics.

Seung-Min, L., Seonnjin, K., \& Park, S. (2009). Self-Paced Sport Events under Temporal Constraints: Visual Search, Quiet Eye, Expertise and Constrained Performance Time in Far Aiming Tasks. International Journal of Applied Sports Sciences, 21(2), 146161.

Tay, S. H. C., Chow, J. Y., Koh, M., \& Button, C. (2012). The effectiveness of keeperindependent penalty kicks using fake visual cues from penalty takers. International Journal of Sport Psychology, 43(5), 403-419.

van der Kamp, J., Rivas, F., van Doorn, H., \& Savelsbergh, G. (2008). Ventral and dorsal contributions in visual anticipation in fast ball sports. International Journal of Sport Psychology, 39, 100-130.

Vickers, J. (2011). Mind over muscle: the role of gaze control, spatial cognition, and the quiet eye in motor expertise. Cognitive Processing, 12(3), 219-222. doi:10.1007/s10339011-0411-2 
1 Vickers. (2007). Perception, cognition, and decision training: The quiet eye in action.

2

3

7

8

9 Champaign, IL: Human Kinetics.

Vickers, J. N. (1996). Visual control when aiming at a far target. Journal of Experimental Psychology: Human Perception and Performance, 22(2), 342-354. doi: $10.1037 / 0096-1523.22 .2 .342$

Vickers, J. N. (1992). Gaze control in putting. Perception, 21(1), 117-132. doi:10.1068/p210117

Vine, S., Moore, L., \& Wilson, M. (2014). Quiet eye training: the acquisition, refinement and resilient performance of targeting skills. European Journal of Sport Science, 14 Suppl 1S235-S242. doi:10.1080/17461391.2012.683815

Wiedman, D. (2006). Bowling: Steps to success. Champaign, IL: Human Kinetics.

Williams, A.M., Davids, K. \& William J.G. (1999). Visual Perception and Action in Sport, London, E \& FN Spon.

Williams, A. M., Singer, R. N., \& Frehlich, S. G. (2002). Quiet eye duration, expertise, and task complexity in near and far aiming tasks. Journal of Motor Behavior, 34(2), 197207. doi:10.1080/00222890209601941

Wilson, M. R., \& Pearcy, R. C. (2009). Visuomotor control of straight and breaking golf putts. Perceptual and Motor Skills, 109(2), 555-562. 
Table 1: Descriptive statistics of performance outcome (\%) for expert and novice for all conditions

\begin{tabular}{llllc}
\hline & \multicolumn{2}{c}{ Novice } & \multicolumn{2}{c}{ Expert } \\
\hline Easy (1-pin) & $M$ & $S D$ & $M$ & $S D$ \\
Hard (10-pin) & 33.3 & 18.6 & 88.3 & 16.0 \\
\hline Overall & 18.3 & 9.8 & 85.0 & 8.4 \\
\hline$* p<.05$ & 25.8 & 4.1 & 86.7 & 4.1 \\
\hline
\end{tabular}


Table 2: Descriptive statistics of quiet eye duration of both skill groups based on performance outcome for all conditions under both methods of analysis

\begin{tabular}{lccccccccc}
\hline & \multicolumn{3}{c}{ Successful } & \multicolumn{5}{c}{ Unsuccessful } \\
\hline & \multicolumn{2}{c}{ Zero value } & \multicolumn{2}{c}{ Trial exclusion } & \multicolumn{2}{c}{ Zero value } & \multicolumn{2}{c}{ Trial exclusion } \\
& $M$ & $S D$ & $M$ & $S D$ & $M$ & $S D$ & $M$ & $S D$ \\
\hline Novice & & & & & & & & \\
Easy (1-pin) & 201.92 & 54.1 & 208.08 & 48.5 & 149.63 & 60.7 & 171.46 & 38.7 \\
Hard (10-pin) & 107.78 & 82.7 & 152.33 & 44.7 & 152.64 & 29.6 & 164.18 & 31.2 \\
& & & & & & & & & \\
\hline Expert & & & & & & & & & \\
Easy (1-pin) & 195.03 & 52.4 & 195.03 & 52.4 & 116.67 & 140.1 & 116.67 & 140.1 \\
Hard (10-pin) & 183.50 & 23.7 & 183.50 & 23.7 & 198.33 & 221.4 & 198.33 & 221.4 \\
& & & & & & & & \\
\hline
\end{tabular}


Table 3: Descriptive statistics of perceived task difficulty and confidence levels and their differences for both groups and all conditions

\begin{tabular}{|c|c|c|c|c|c|c|}
\hline & \multicolumn{2}{|c|}{$\begin{array}{l}\text { Perceived } \\
\text { difficulty }\end{array}$} & \multicolumn{2}{|c|}{ Confidence level } & \multicolumn{2}{|c|}{$\begin{array}{l}\text { Difference } \\
\text { (difficulty- } \\
\text { confidence) }\end{array}$} \\
\hline & $M$ & $S D$ & $M$ & $S D$ & $M$ & $S D$ \\
\hline \multicolumn{7}{|l|}{ Novice } \\
\hline Easy (1-pin) & 5.20 & 1.23 & 2.90 & 0.86 & 2.30 & 2.07 \\
\hline Hard (10-pin) & 6.03 & 0.85 & 2.43 & 0.52 & 3.60 & 1.11 \\
\hline \multicolumn{7}{|l|}{ Expert } \\
\hline Easy (1-pin) & 2.17 & 0.76 & 5.85 & 0.78 & -3.70 & 1.57 \\
\hline Hard (10-pin) & 1.95 & 0.85 & 5.97 & 0.83 & -4.02 & 1.61 \\
\hline
\end{tabular}


Figure Captions

Figure 1. Arrangement of pins.

$\begin{array}{ccccc}7 & 8 & & 9 & 10 \\ 4 & & 5 & & 6\end{array}$


Figure 2. Overview of experimental set-up (top view) used in the present study.

\section{Pins}
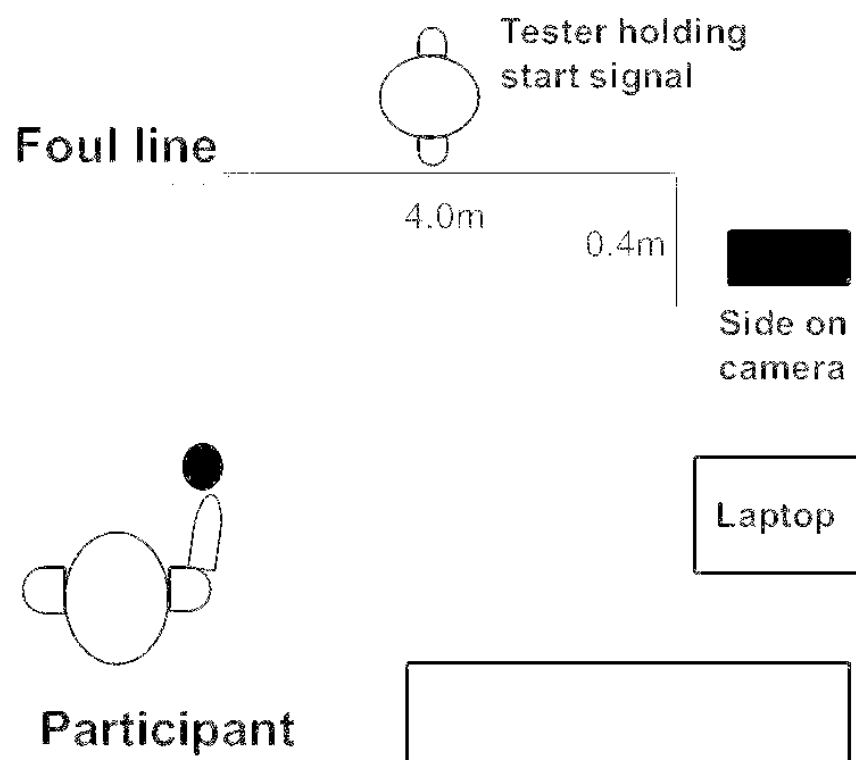

Laptop

Rest Area 
Figure 3a. Mean QE duration (ms) for easy condition by individual participants (novice: ID 1-6, expert: ID: 7-12) with mean and error bar represented by a bold horizontal line and shaded rectangle. Trials with absent QE durations were plotted as the zero value point.

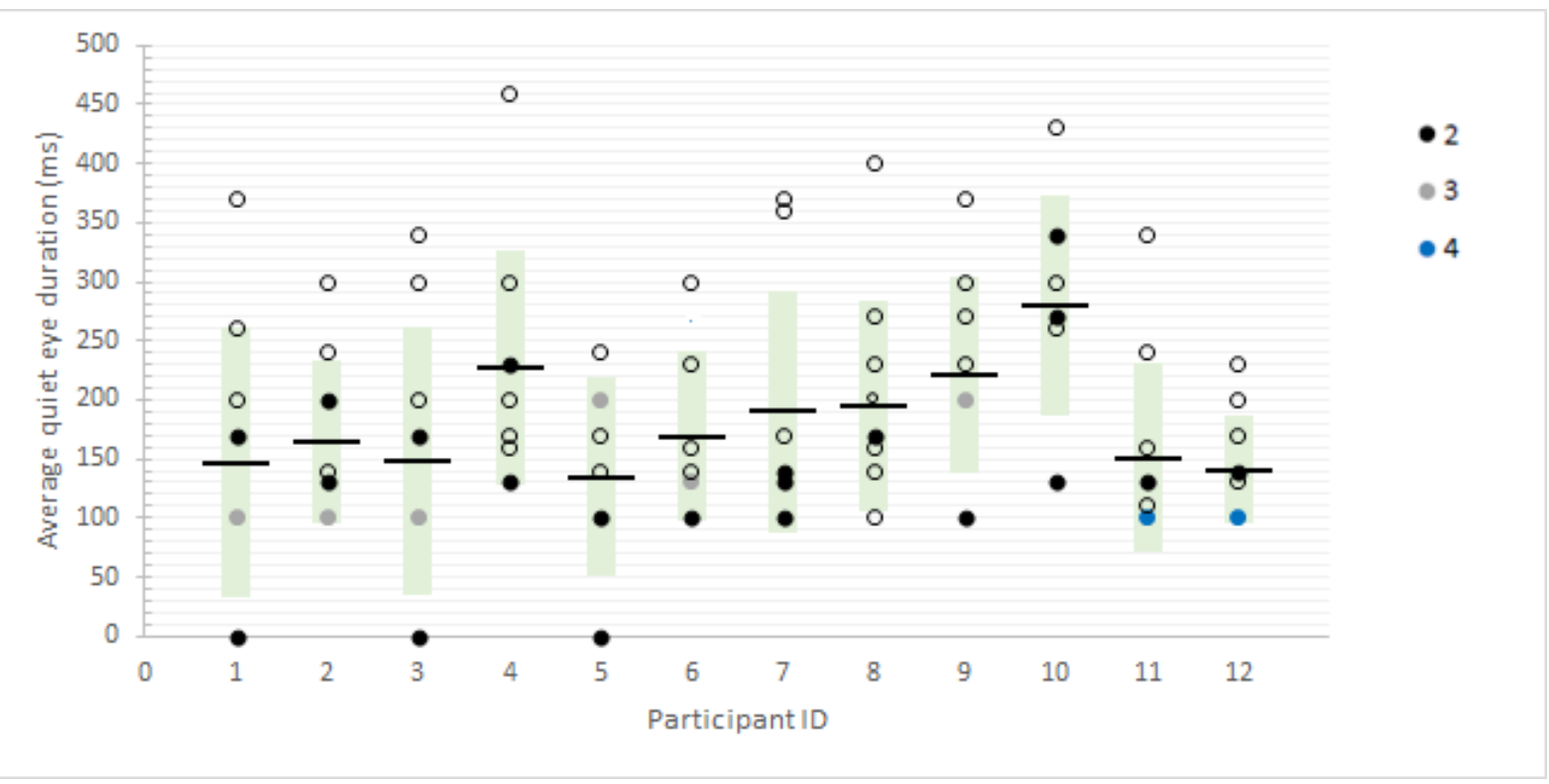


1 Figure 3b. Mean QE duration (ms) for hard condition by individual participants (novice: ID 2 1-6, expert: ID: 7-12) with mean and error bar represented by a bold horizontal line and 3 shaded rectangle. Trials with absent QE durations were plotted as the zero value point.

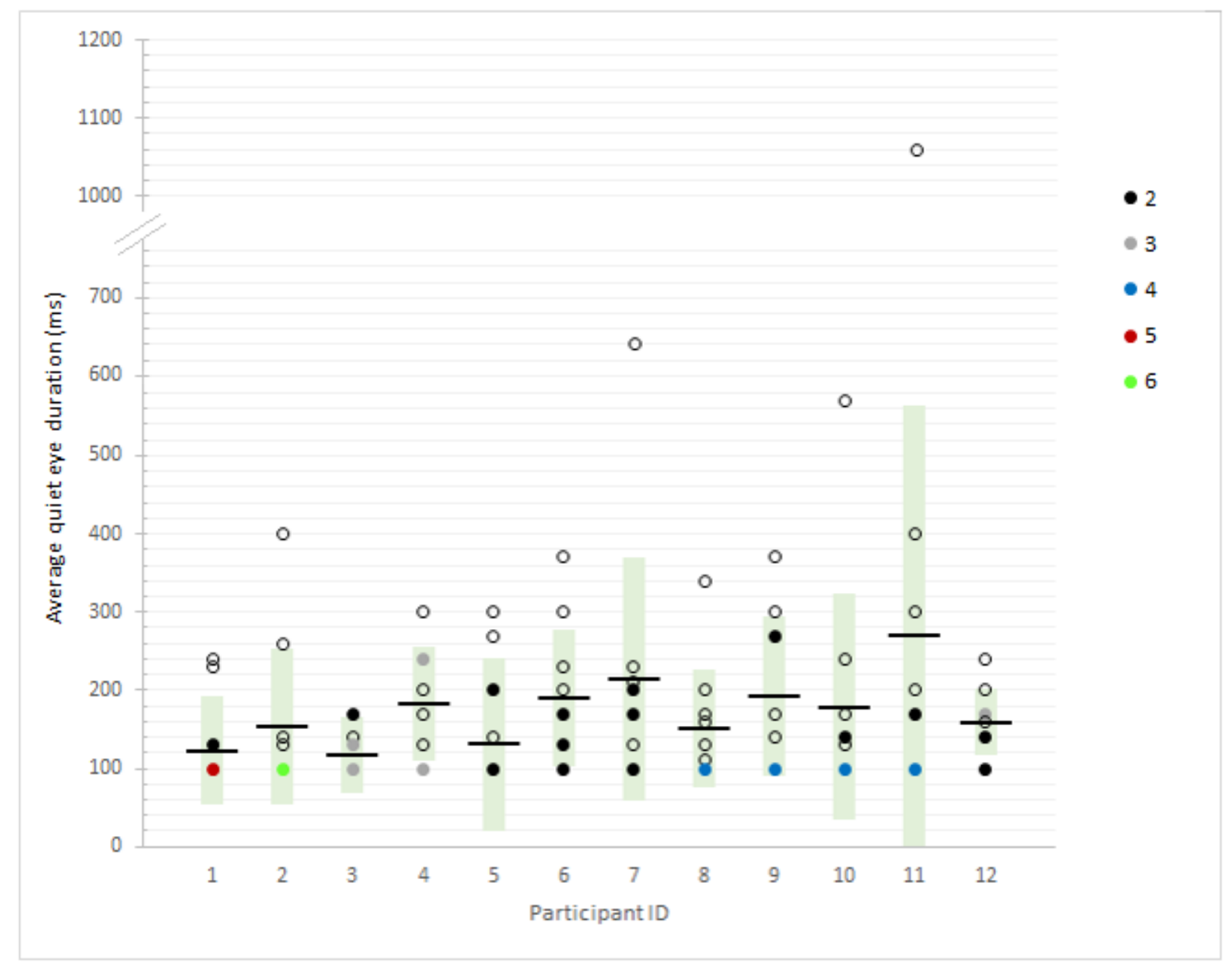


Figure 4. Performance outcome scores $(\%)$ for both easy and hard conditions for all participants.

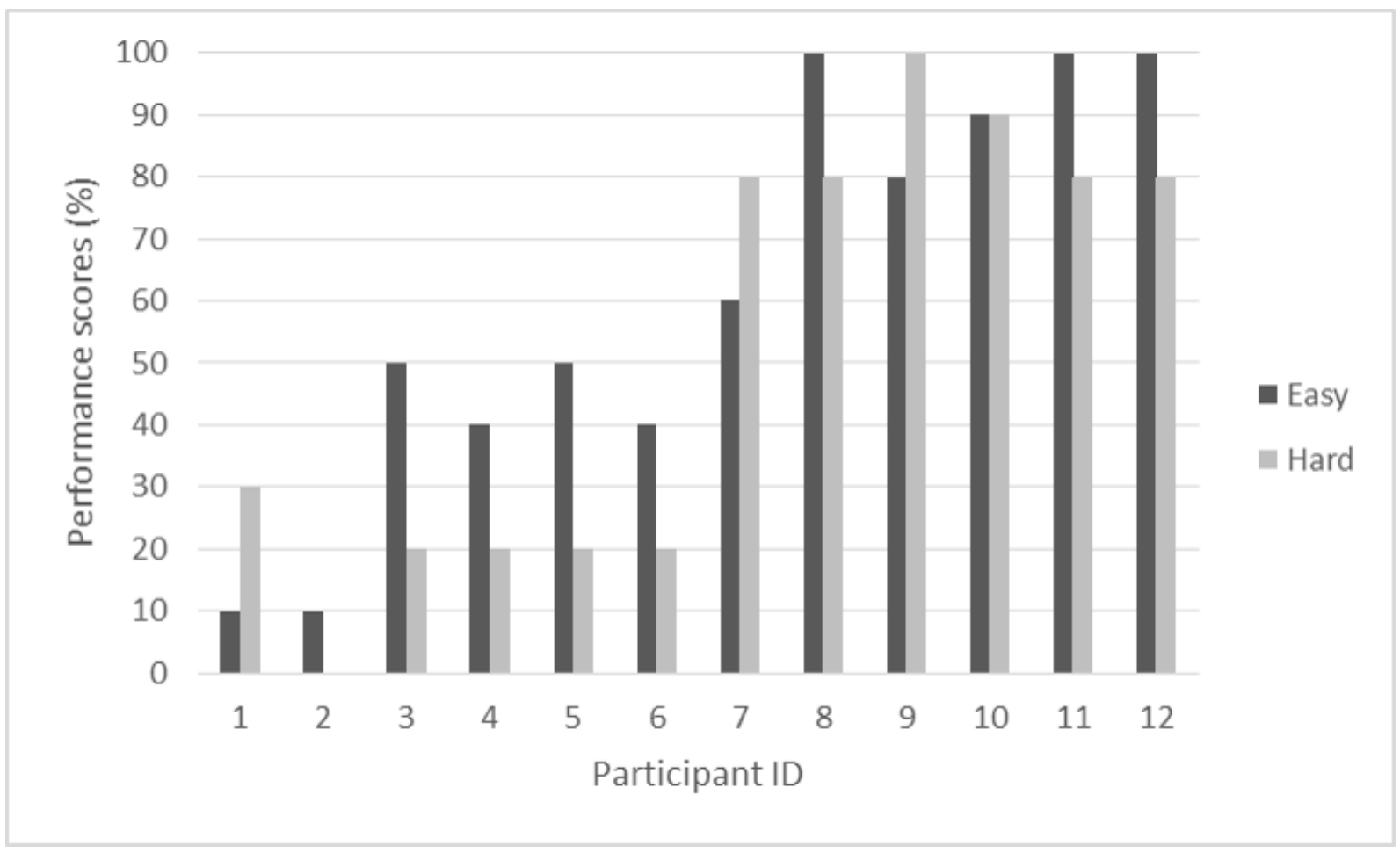


Figure 5a. Mean QE duration (ms) between hits and misses for the easy condition by novice participants (novice: ID 1-6, expert: ID: 7-12).

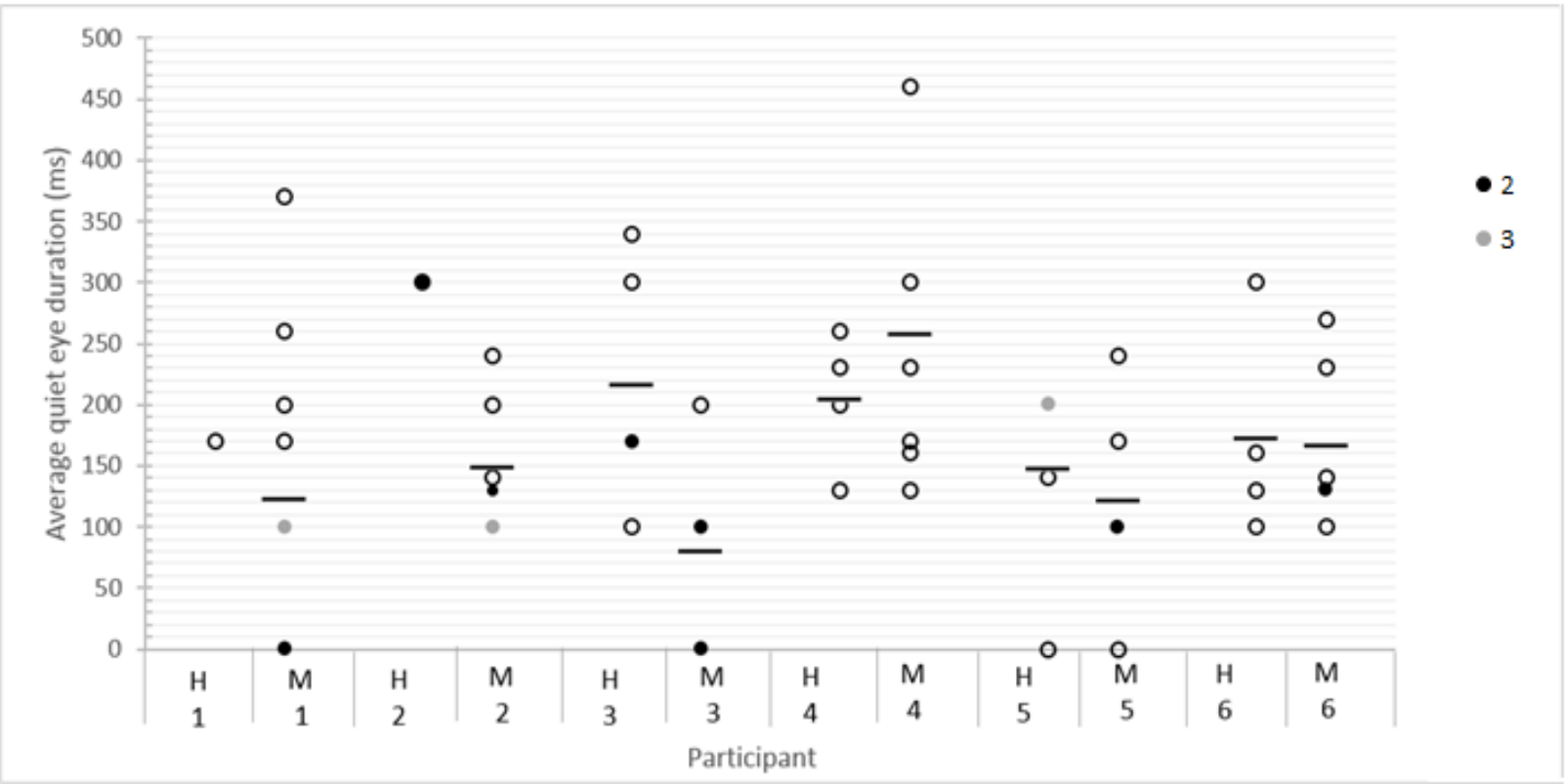


Figure 5b. Mean QE duration (ms) between hits and misses for the hard condition by novice participants.

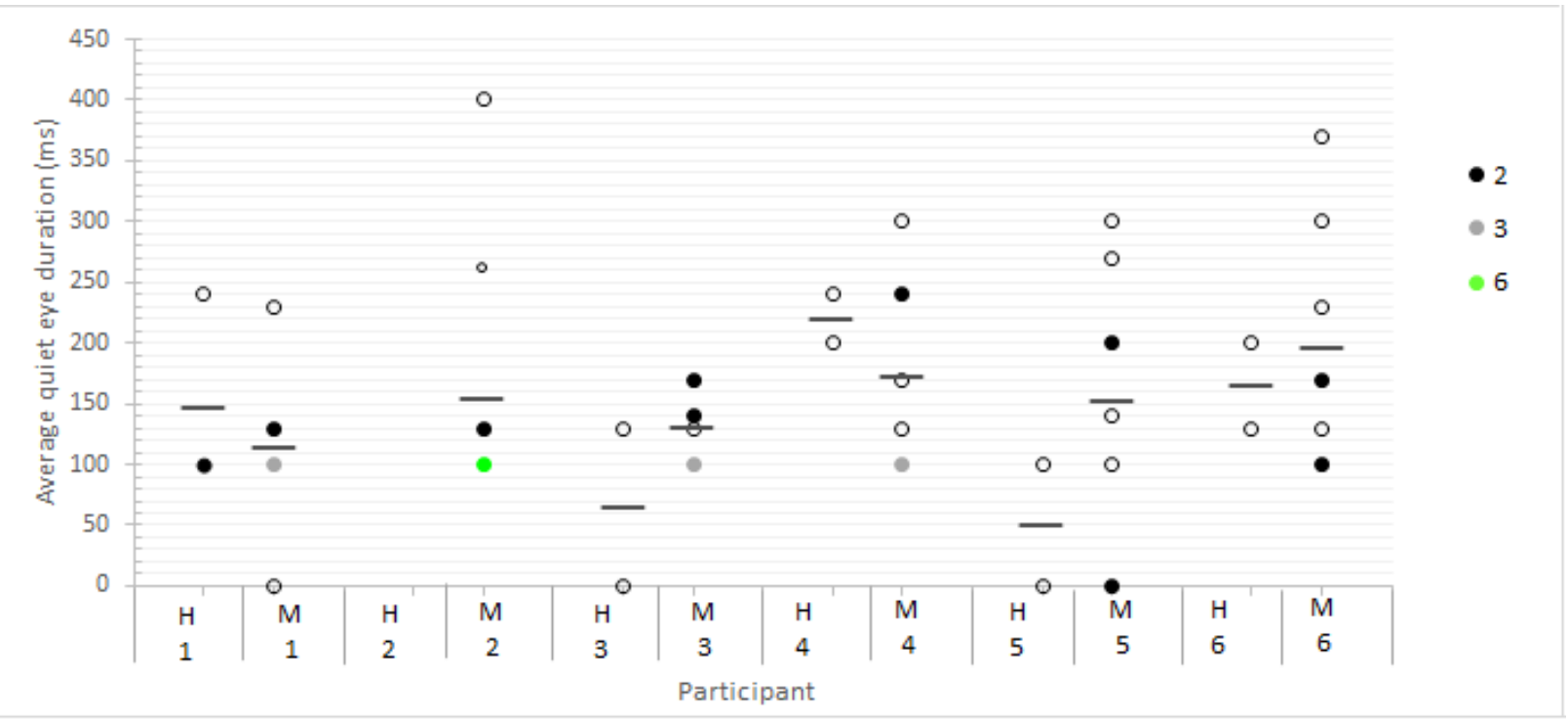

